Health Place. 2012 January ; 18(1): 96-99. doi:10.1016/j.healthplace.2011.08.010.

\title{
Exploring walking differences by socioeconomic status using a spatial agent-based model
}

\author{
Yong Yang, PhD, \\ Department of Epidemiology, Center for Social Epidemiology and Population Health, University of \\ Michigan, Ann Arbor, Michigan, USA
}

Ana V. Diez Roux, PhD, MD,

Department of Epidemiology, Center for Social Epidemiology and Population Health, University of Michigan, Ann Arbor, Michigan, USA

\begin{abstract}
Amy H. Auchincloss, PhD,
Department of Epidemiology and Biostatistics, Drexel University, Philadelphia, Pennsylvania, USA
\end{abstract}

Daniel A. Rodriguez, PhD, and

Department of City and Regional Planning, University of North Carolina, Chapel Hill, North, Carolina, USA

Daniel G. Brown, PhD

School of Natural Resources \& Environment, University of Michigan, Ann Arbor, Michigan, USA

\begin{abstract}
We use an exploratory agent-based model of adults' walking behavior within a city to examine the possible impact of interventions on socioeconomic differences in walking. Simulated results show that for persons of low socioeconomic status, increases in walking resulting from increases in their positive attitude towards walking may diminish over time if other features of the environment are not conducive to walking. Similarly, improving the safety level for the lower SES neighborhoods may be effective in increasing walking, however, the magnitude of its effectiveness varies by levels of land use mix, such that effects of safety are greatest when persons live in areas with a large mix of uses.
\end{abstract}

\section{Keywords \\ agent-based model; walking; socioeconomic status}

\section{Introduction}

It is well established that lower socioeconomic status (SES) is associated with lower levels of physical activity (Ford et al., 1991; Gidlow et al., 2006; Mackenbach et al., 2008).

(C) 2011 Elsevier Ltd. All rights reserved.

Corresponding author's name and complete contact information (mailing address, telephone and fax numbers, and e-mail address): Yong Yang, Department of Epidemiology, Center for Social Epidemiology and Population Health, University of Michigan, Ann Arbor, MI, 48109, USA, Telephone: 734-615-9211, Fax: 734-763-5706, yongyang@ umich.edu.

Publisher's Disclaimer: This is a PDF file of an unedited manuscript that has been accepted for publication. As a service to our customers we are providing this early version of the manuscript. The manuscript will undergo copyediting, typesetting, and review of the resulting proof before it is published in its final citable form. Please note that during the production process errors may be discovered which could affect the content, and all legal disclaimers that apply to the journal pertain. 
Walking is increasingly recognized as an important component of physical activity which can be easily incorporated into daily life, and is potentially amenable to interventions. Among US urban adults, lower SES persons tend to walk more for transportation and walk less for recreation than higher SES persons (Besser and Dannenberg, 2005; Kruger et al., 2008; Pucher and Renne, 2003). SES differences in walking are likely to emerge from the dynamic interplay of individual and environmental factors (Ball et al., 2007; Cerin et al., 2009; Kamphuis et al., 2009). A better understanding of the factors driving SES differences in walking could shed light on strategies not only to reduce SES disparities in physical activity but also to improve population levels of walking generally.

Agent-based models (ABMs) are computational models that can be used to simulate the dynamic interactions of agents and environment in order to gain understanding of the functioning of the system (Axtell and Epstein, 1994; Bonabeau, 2002). An advantage of ABMs over statistical models is they account for feedbacks between agents and between agents and environments over time. It has been argued that ABMs may be of use in the investigation of how dynamic processes shape the distribution of health outcomes (Auchincloss and Diez Roux, 2008; Galea et al., 2009).

We use a previously developed exploratory ABM of adults' walking behavior (Yang et al., 2011) to examine the possible impact of interventions on socioeconomic differences in overall walking and in walking for different purposes. We examine the impact of combinations of two strategies commonly proposed as ways to increase walking, and their interactions: improving people's attitudes towards walking (an individual-targeted intervention, for example through health education campaigns) and improving safety (a social environment intervention, for example through community policing efforts). In order to further illustrate how the model can be used to explore interactions between different types of interventions, we explore whether the impact of these is modified by mixed land use (a feature of the physical environment).

This study's intent was to assess possible impacts of certain types of interventions rather than to make specific predictions. We use a simplistic exploratory model and like all models, conclusions are contingent on assumptions encoded in the model. However, we believe our assumptions are reasonable based on current knowledge and the model replicates key patterns observed in the real world.

\section{Research method}

Details of the model are provided elsewhere (Yang et al., 2011). Briefly, the model represents a hypothetical city of 108,000 adults and locations are placed within a grid space. Each individual is assigned properties such as SES, attitude towards walking, home and work location. Individuals perform activities such as traveling to work, for shopping, and for recreation. Whether an individual walks is a function of distance to different activities, walking ability, and attitude towards walking. An individual's attitude towards walking evolves over time through several feedbacks including prior walking experiences (e.g., the safety level of the walking route and number of walking trips that cross the walking route), and attitudes of the other individuals within her/his social network. Parameters such as probabilities and maximum walking distances for different activities were calibrated using data from 2001 National Household Travel Survey (NHTS, 2001)

Previous work (Yang et al., 2011) identified one scenario which generated SES differences in walking that were relatively consistent with the ones most commonly observed in empirical data (Besser and Dannenberg, 2005; Kruger et al., 2008; Pucher and Renne, 2003). In this scenario (subsequently referred to as the baseline scenario), the city is divided into five concentric zones. There is perfect segregation by SES, such that the lowest SES 
residents live in the core (Zone 1) and the SES of residents increases from the core to the periphery (Zone 5). The density of non-residential locations such as worksites or amenities for shopping and recreation decays outwards with the ratio of 0.5 from one zone to another. Safety levels increase outwards from Zone 1 (lowest safety value of 1) to Zone 5 (highest safety value of 5). Unless noted, at inception the model assumes no differences in attitude towards walking across SES groups.

We compared a total of 12 scenarios labeled BSx, ASx, ESx and DSx where x can be 1, 3 or 5. BS1 (B for baseline) is the baseline scenario described above. BS3 and BS5 modify the safety level of the core area (zone 1) only: in BS3 the safety level is increased to an intermediate level (value $=3$ ) and in BS5 the safety level is increased to the highest level (value=5). Scenarios ASx (A for attitude) are similar to BSx, except that the attitude towards walking of the lowest SES persons (SES1) is increased so that at inception, the lowest SES residents have a preference for walking that is twice as high as the other SES groups. Because the mean walking attitude for the population as a whole is assumed to be constant, this implied that the attitude for the other SES groups was decreased slightly. In order to further examine how land use modified the impacts of changes in safety we also developed scenarios ESx (E for even) and DSx (D for decay) which modify the land-use distribution of the baseline scenario: in ESx non-residential density is assumed to be even across zones; in DSx the density of non-residential uses decays outwards with a ratio of $1 / 3$ in order to create more pronounced differences in land use than in the baseline scenarios.

Results are based on the values measured on day 10,000 and averaged across 10 simulations. Day 10,000 was selected because prior work (Yang et al., 2011) suggested that SES differences in walking had achieved an approximate equilibrium after this point.

\section{Results}

Figure 1 shows total walking trips per person per day, and Figure 2 shows walking trips per person per day for different purposes: for work, for basic needs (including grocery and other shopping and visiting social places) and for recreation.

In the baseline scenario BS1 (upper left, Figure 1), the mean number of daily walking trips per person was about $0.25-0.26$ and was not patterned by SES. Increasing safety in the lowest SES areas, however, substantially changed this pattern such that the lowest SES persons walked substantially more than higher SES persons in scenario BS3 and BS5 (compare across first row of Figure 1). In contrast, increasing the attitude towards walking in the lowest SES groups had no impact (compare first and second rows in Figure 1). When non-residential densities were evenly distributed (third row, Figure 1) the lower SES groups walked less than the higher SES groups, but SES differences were reduced when safety in the core (Zone 1) was high (compare ES3, ES5 to ES1 row three Figure 1). More extreme differences in land-use further accentuated SES differences in walking observed in the BS scenarios (compare top and bottom rows, Figure 1), i.e., lowest SES groups walked substantially more than higher SES groups even in the DS1 scenario).

The impact of improving safety in the lowest SES areas on increases in walking in the lowest SES group was greater when land-use mix was very unequally distributed (bottom row, Figure 1) and smaller when land-use mix was evenly distributed (third row, Figure 1). Analogously, increasing mixed land use in the core resulted in substantial increases in walking among the lowest SES group, and this effect was greatest when safety in the core was high (compare walking for low SES groups across rows 3 (no differences in mixed use) and 4 (extreme differences in mixed use) for the leftmost and rightmost columns). 
The patterns observed for walking for basic needs were very similar to those observed for total walking (Figure $2 \mathrm{~b}$ ) partly because walking for basic needs represents a substantial share of all walking trips. However, important differences were observed for walking to work and walking for recreation. Lower SES persons walked more to work than higher SES persons across all scenarios although the SES gradients were substantially weaker and almost absent when land-use mix was evenly distributed (Figure 2a, third row). Walking for recreation was consistently higher in the higher SES groups than in the lower SES groups, but this gradient became less pronounced as safety in the low SES areas increased (Figure $2 \mathrm{c}$, compare across columns). Differences in land-use had virtually no impact on walking for recreation (compare rows 3 and 4 of figure $2 \mathrm{c}$ ).

Figure 2 also shows that increasing attitude towards walking in the lower SES groups had no impact on any type of walking (compare first and second rows). Increasing safety in the core increased walking in the lowest SES groups for all scenarios and all types of walking (compare across columns). However effects of increases in safety on walking to work and for basic needs were largest when mixed land use in the core was also high (bottom row figures $2 a$ and $2 b)$.

\section{Discussion}

The simulation results suggest that the walking increases resulting from increasing a positive attitude towards walking diminish over time if other features of the environment (such as safety) are not conducive to walking. This is consistent with research suggesting that the long-term maintenance of the effects of individual-based physical activity interventions is challenging (Müller-Riemenschneider et al., 2008). Another finding is that although increasing the safety level for lower SES neighborhoods may be effective in increasing walking, the magnitude of its effectiveness varies by the patterns of land-use mix.

In the baseline scenario, attitudes towards walking were equal across SES groups at inception. However, the positive attitude towards walking decreased in the lower SES persons but increased in the higher SES persons over time. This occurred because of both the differential exposure to safety by SES and the feedbacks from walking experiences to walking attitude. Any SES differentials in attitude at inception were overcome by these feedback mechanisms operating over time.

In addition, safety and land-use mix acted synergistically such that the effect on walking among lower SES groups of one was magnified in the presence of the other. When the concentration of non-residential uses in the core was low the chance that a low SES person would find a suitable location within walking distance was also low, and the effect of safety on walking was diminished because the probability that persons would be exposed to the higher safety through walking was lower, hence the feedback to attitude had a lower chance to operate. The opposite occurred when mixed land use was high in low SES areas.

It is important to note that these simulations are based on a stylized exploratory model and are meant to illustrate the types of insights that can be obtained from these approaches. Drawing reliable conclusions about the predicted impact of interventions in a particular setting would require many model refinements and incorporation of additional data to support the assumptions in the model. Whenever possible, conclusions regarding impacts of interventions should be confirmed in experimental designs. In this exploratory model, landuse mix and safety were selected as examples of features of the built and social environments which have been linked to walking in prior work (Yang et al., 2011). Many other factors and dynamics that may contribute to walking were not included. Future modifications can include, for example, incorporation of a public transportation system as 
well as the inclusion of long-term influences of walking patterns on subsequent changes in the built and social environments.

A major challenge is making these models empirically grounded given that data to support some parameters may be unavailable or may be impossible to obtain. Nevertheless they can yield insights into basic dynamics that are not obtainable using other approaches. Together with other methods, including observational studies as well as quasi experiments and trials, ABMs may contribute to providing the evidence base necessary to improve population levels of walking and reduce inequalities in walking.

\section{References}

Auchincloss AH, Diez Roux AV. A new tool for epidemiology: the usefulness of dynamic-agent models in understanding place effects on health. American Journal of Epidemiology. 2008; 168:18. [PubMed: 18480064]

Axtell, R.; Epstein, JM. The Bulletin of The Santa Fe Institute. 1994 Winter. Agent-Based Modeling: Understanding Our Creations; p. 28-32.

Ball K, Timperio A, Salmon J, Giles-Corti B, Roberts R, Crawford D. Personal, social and environmental determinants of educational inequalities in walking: a multilevel study. Journal of Epidemiology and Community Health. 2007; 61:108-114. [PubMed: 17234868]

Besser LM, Dannenberg AL. Walking to Public Transit Steps to Help Meet Physical Activity Recommendations. American Journal of Preventive Medicine. 2005; 29:273-280. [PubMed: 16242589]

Bonabeau E. Agent-based modeling: Methods and techniques for simulating human systems. PNAS. 2002; 99:7280-7287. [PubMed: 12011407]

Cerin E, Leslie E, Owen N. Explaining socio-economic status differences in walking for transport: An ecological analysis of individual, social and environmental factors. Social Science \& Medicine. 2009; 68:1013-1020. [PubMed: 19193480]

Ford ES, Merritt RK, Heath GW, Powell KE, Washburn RA, Kriska A, Haile G. Physical Activity Behaviors in Lower and Higher Socioeconomic Status Populations. American Journal of Epidemiology. 1991; 133:1246-1256. [PubMed: 2063832]

Galea S, Riddle M, Kaplan GA. Causal thinking and complex system approaches in epidemiology. Int J Epidemiol. 2009:dyp296.

Gidlow C, Johnston LH, Crone D, Ellis N, James D. A systematic review of the relationship between socio-economic position and physical activity. Health Education Journal. 2006; 65:338-367.

Kamphuis CB, Lenthe FJv, Giskes K, Huisman M, Brug J, Mackenbach JP. Socioeconomic differences in lack of recreational walking among older adults: the role of neighbourhood and individual factors. International Journal of Behavioral Nutrition and Physical Activity. 2009:6. [PubMed: 19159476]

Kruger J, Ham SA, Berrigan D, Ballard-Barbash R. Prevalence of transportation and leisure walking among U.S. adults. Preventive Medicine. 2008; 47:329-334. [PubMed: 18445507]

Mackenbach J, Stirbu I, Roskam A. Socioeconomic inequalities in health in 22 European countries. New England Journal of Medicine. 2008; 358:2486-2481.

Müller-Riemenschneider F, Reinhold T, Nocon M, Willich SN. Long-term effectiveness of interventions promoting physical activity: A systematic review. Preventive Medicine. 2008; 47:354-368. [PubMed: 18675845]

NHTS. 2001 National Household Travel Survey. 2001.

Pucher J, Renne JL. Socioeconomics of Urban Travel: Evidence from the 2001 NHTS. Transportation Quarterly. 2003; 57:49-77.

Yang Y, Diez Roux AV, Auchincloss AH, Rodriguez DA, Brown DG. A spatial agent-based model for the simulation of adults' daily walking within a city. American Journal of Preventive Medicine. 2011; 40:353-361. [PubMed: 21335269] 


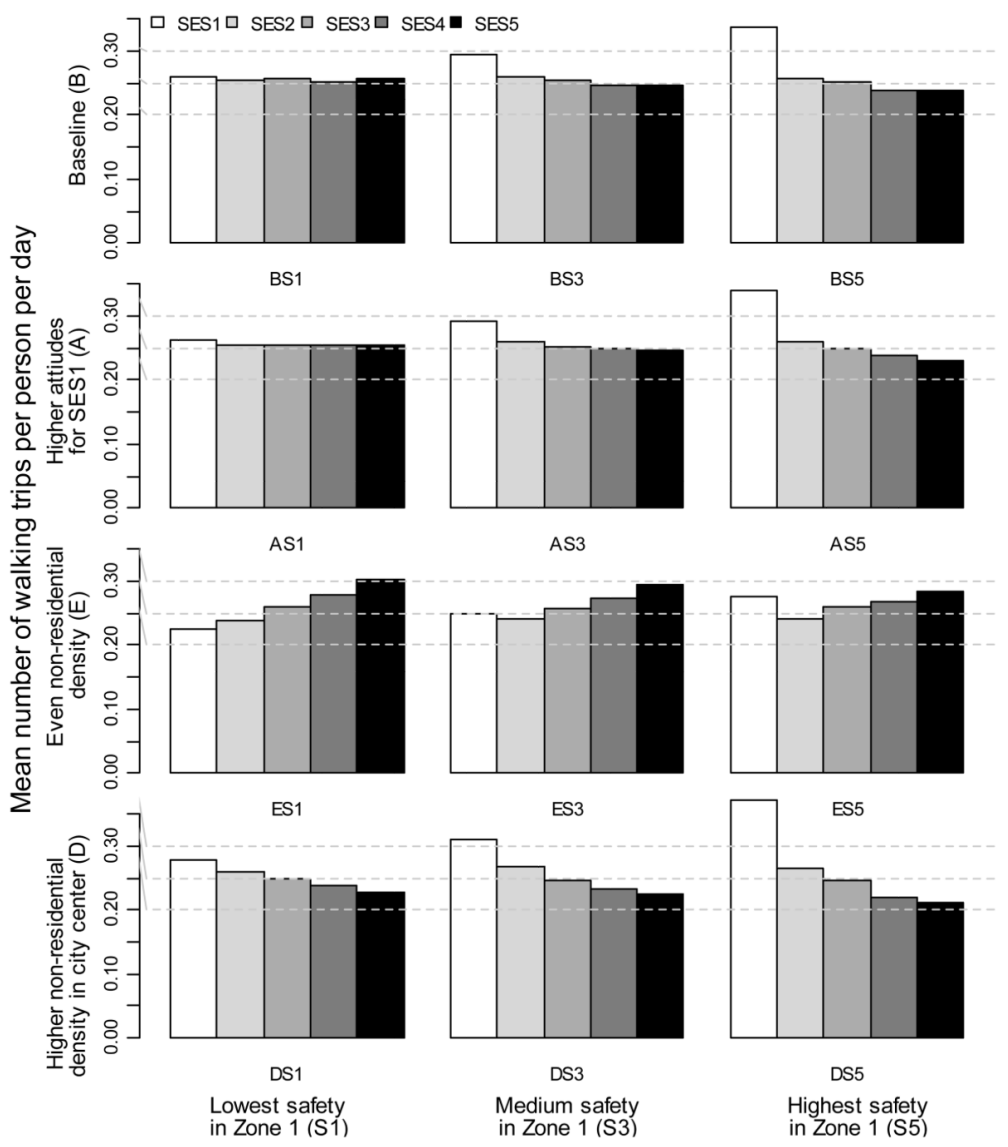

Figure 1.

Mean number of walking trips per person per day for five socio-economic status (SES) groups for various scenarios as described in the text. 

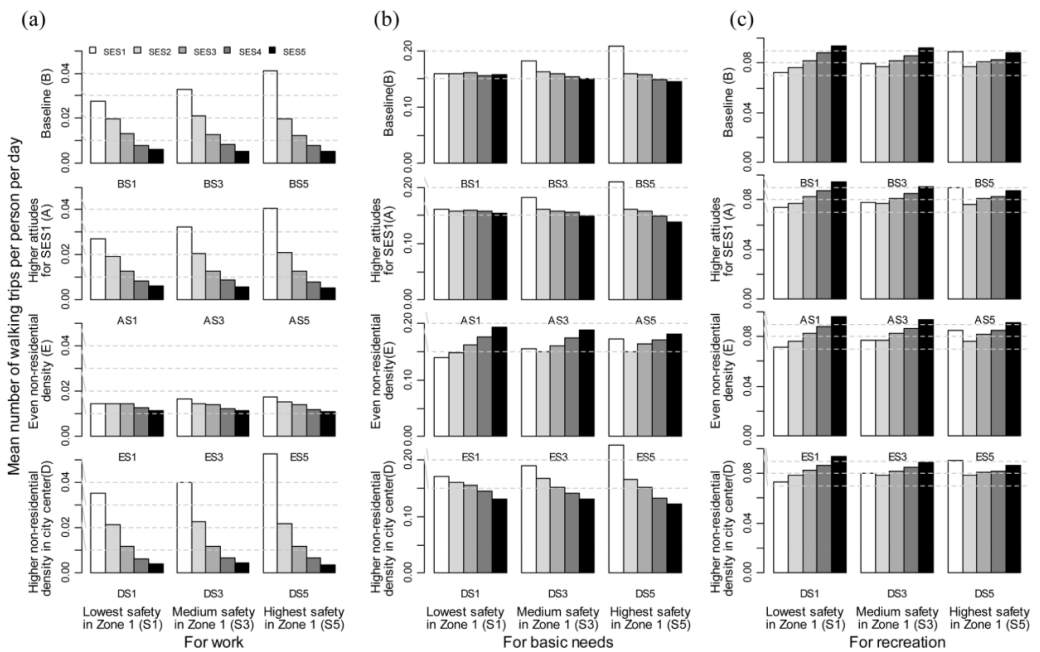

Figure 2.

Mean number of walking trips per person per day to work (a), for basic needs (b) and for recreation (c) for five socio-economic status (SES) groups for different scenarios as described in the text. 